\title{
ABOUT A TWIN SOLUTION OF THE VEKUA EQUATION
}

\author{
Slagjana Brsakoska
}

\begin{abstract}
In the paper main object of research is the Vekua equation. Two types of functions are found that are strongly connected to each other because it will be proven that if one of them is a solution of the Vekua equation, so will be the other one with a corresponding condition. Three different cases are considered.
\end{abstract}

\section{INTRODUCTION}

G. V. Kolosov in 1909 [1], when he was solving a problem from the theory of elasticity, introduced the expressions

$$
\begin{array}{ll}
\frac{1}{2}\left[\frac{\partial u}{\partial x}+\frac{\partial v}{\partial y}+i\left(\frac{\partial v}{\partial x}-\frac{\partial u}{\partial y}\right)=\frac{\hat{d} W}{d z} \quad\right. \text { and } \\
\frac{1}{2}\left[\frac{\partial u}{\partial x}-\frac{\partial v}{\partial y}+i\left(\frac{\partial v}{\partial x}+\frac{\partial u}{\partial y}\right)=\frac{\hat{d} W}{d \bar{z}}\right.
\end{array}
$$

known as operatory derivatives of a complex function $W=W(z)=u(x, y)$ $+i v(x, y)$ from a complex variable $z=x+i y$ and $\bar{z}=x-i y$, respectively. The operator rules for these derivatives are given in the monograph of $\Gamma$. $\mathrm{H}$. Положий [2] (pages 18-31). In the mentioned monograph, are also defined the so called operatory integrals

$$
\hat{\int} f(z) d z \text { and } \hat{\int} f(z) d \bar{z}
$$

by $z=x+i y$ and $\bar{z}=x-i y$, respectively, from the complex function $f=f(z)$ in the area $D \subseteq \mathbb{C}$, where their operatory rules are proven as well, page $32-41$.

2010 Mathematics Subject Classification. 34M45, 35Q74.

Key words and phrases. areolar derivative, areolar equation, solution, analytic function, Vekua equation. 


\section{FORMULATION OF THE PROBLEM}

Main object of this research is the Vekua equation

$$
\frac{\hat{d} W}{d \bar{z}}=A \bar{W}+B W+F
$$

where the functions $A=A(z), B=B(z), F=F(z)$ are arbitrary functions from complex variable without any limitation or condition that they have to fulfill.

Because in general case there is no method for finding its general solution, we explore the idea to find some solution of the Vekua equation (1) in the following form:

$$
W=W(\varphi(\bar{z}), \psi(z))
$$

where $\varphi=\varphi(\bar{z})$ is antyanalytic function and $\psi=\psi(z)$ is analytic function.

\section{Main Result}

Case 1. Let $W=\frac{\varphi(\bar{z})}{\psi(z)}$ be a solution of the equation (1) (and is from the form (2)), i.e. it is a ratio from one antyanalytic and one analytic function. That means that this function satisfies the equation (1), so if we find the operator derivative by $\bar{z}$ from $W$ and replace it in (1) we get:

$$
\begin{aligned}
& \frac{\hat{d} W}{d \bar{z}}=\frac{\hat{d}}{d \bar{z}}\left(\frac{\varphi(\bar{z})}{\psi(z)}\right)=\frac{1}{\psi(z)} \cdot \frac{\hat{d} \varphi(\bar{z})}{d \bar{z}} \\
& \frac{1}{\psi(z)} \cdot \frac{\hat{d} \varphi(\bar{z})}{d \bar{z}}=A\left(\frac{\varphi(\bar{z})}{\psi(z)}\right)+B\left(\frac{\varphi(\bar{z})}{\psi(z)}\right)+F \\
& \bar{\psi}(\bar{z}) \cdot \frac{\hat{d} \varphi(\bar{z})}{d \bar{z}}=A \bar{\varphi}(z) \psi(z)+B \varphi(\bar{z}) \bar{\psi}(\bar{z})+F \psi(z) \bar{\psi}(\bar{z})
\end{aligned}
$$

If we make one more transformation, we can get a proof to one more interesting statement. If we add on both sides the expression $\varphi(\bar{z}) \cdot \frac{\hat{d} \bar{\psi}(\bar{z})}{d \bar{z}}$ and given into consideration that

$$
\frac{\hat{d}(\bar{\psi}(\bar{z}) \cdot \varphi(\bar{z}))}{d \bar{z}}=\frac{\hat{d} \bar{\psi}(\bar{z})}{d \bar{z}} \cdot \varphi(\bar{z})+\bar{\psi}(\bar{z}) \cdot \frac{\hat{d} \varphi(\bar{z})}{d \bar{z}}
$$

then, we get

$$
\frac{\hat{d}(\bar{\psi}(\bar{z}) \cdot \varphi(\bar{z}))}{d \bar{z}}=\frac{\hat{d} \bar{\psi}(\bar{z})}{d \bar{z}} \cdot \varphi(\bar{z})+A \overline{(\varphi(\bar{z}) \bar{\psi}(\bar{z}))}+B \cdot \varphi(\bar{z}) \bar{\psi}(\bar{z})+F \psi(z) \bar{\psi}(\bar{z})
$$

So, if 


$$
\psi(z) \bar{\psi}(\bar{z})=1 \text { and } \varphi(\bar{z}) \cdot \frac{\hat{d} \bar{\psi}(\bar{z})}{d \bar{z}}=0
$$

we get another solution to the Vekua equation (1), i.e. the function $W_{1}=\varphi(\bar{z}) \cdot \bar{\psi}(\bar{z})$ which is not from the form (2). It is an antyanalytic function. If we want a solution that $W \neq 0$, then $\varphi(\bar{z}) \neq 0$, which means that the second condition in (3) is $\frac{\hat{d} \bar{\psi}(\bar{z})}{d \bar{z}}=0$.

So, now we can formulate the proven fact as a theorem.

Theorem 1. Let $\varphi=\varphi(\bar{z})$ be an antyanalytic function and $\psi=\psi(z)$ be an analytic function. If $W=\frac{\varphi(\bar{z})}{\psi(z)}$ is a solution to the Vekua equation (1), then $W_{1}=\varphi(\bar{z}) \bar{\psi}(\bar{z})$ is also a solution to the Vekua equation (1), if the conditions (3) are satisfied.

Case 2. Let $W=\frac{\psi(z)}{\varphi(\bar{z})}$ be a solution of the equation (1) (and is from the form (2)), i.e. it is a ratio from one analytic and one antyanalytic function. That means that this function satisfies the equation (1), so if we find the operator derivative by $\bar{z}$ from $W$ and replace it in (1) we get:

$$
\begin{aligned}
& \frac{\hat{d} W}{d \bar{z}}=\frac{\hat{d}}{d \bar{z}}\left(\frac{\psi(z)}{\varphi(\bar{z})}\right)=\psi(z) \frac{\hat{d}}{d \bar{z}}\left(\frac{1}{\varphi(\bar{z})}\right)=-\frac{\psi(z)}{\varphi^{2}(\bar{z})} \cdot \frac{\hat{d} \varphi(\bar{z})}{d \bar{z}} \\
& -\frac{\psi(z)}{\varphi^{2}(\bar{z})} \cdot \frac{\hat{d} \varphi(\bar{z})}{d \bar{z}}=A\left(\frac{\psi(z)}{\varphi(\bar{z})}\right)+B\left(\frac{\psi(z)}{\varphi(\bar{z})}\right)+F \\
& -\frac{\psi(z)}{\varphi(\bar{z})} \cdot \bar{\varphi}(z) \cdot \frac{\hat{d} \varphi(\bar{z})}{d \bar{z}}=A \bar{\psi}(\bar{z}) \varphi(\bar{z})+B \psi(z) \bar{\varphi}(z)+F \varphi(\bar{z}) \cdot \bar{\varphi}(z)
\end{aligned}
$$

If we make one more transformation, we can get a proof to another interesting statement. Here we expect a new solution of the Vekua equation to be the function $W_{1}=\bar{\varphi}(z) \cdot \psi(z)$. It is analytic function, so its areolar derivative is 0 . So, if we add on both sides the expression $\frac{\hat{d} W_{1}}{d \bar{z}}$, then we get

$$
-\frac{\psi(z)}{\varphi(\bar{z})} \cdot \bar{\varphi}(z) \cdot \frac{\hat{d} \varphi(\bar{z})}{d \bar{z}}+\frac{\hat{d}(\psi(z) \bar{\varphi}(z))}{d \bar{z}}=A(\overline{\psi(z) \bar{\varphi}(z)})+B \psi(z) \bar{\varphi}(z)+F \varphi(\bar{z}) \cdot \bar{\varphi}(z)
$$

So, if 


$$
\varphi(\bar{z}) \cdot \bar{\varphi}(z)=1 \text { and }-\frac{\psi(z)}{\varphi(\bar{z})} \cdot \bar{\varphi}(z) \cdot \frac{\hat{d} \varphi(\bar{z})}{d \bar{z}}=0
$$

we get another solution to the Vekua equation (1), i.e. the function $W_{1}=\bar{\varphi}(z) \cdot \psi(z)$ which is not from the form (2). It is an analytic function. Again, the second condition in (4), means that $\frac{\hat{d} \varphi(\bar{z})}{d \bar{z}}=0$.

So, now we can formulate the proven fact as a theorem.

Theorem 2. Let $\varphi=\varphi(\bar{z})$ be an antyanalytic function and $\psi=\psi(z)$ be an analytic function. If $W=\frac{\psi(z)}{\varphi(\bar{z})}$ is a solution to the Vekua equation (1), then $W_{1}=\bar{\varphi}(z) \cdot \psi(z)$ is also a solution to the Vekua equation (1), if the conditions (4) are satisfied.

Case 3. Let $W=\psi(z) \varphi(\bar{z})$ is a solution of the equation (1) (and is from the form (2)), i.e. it is a product from one antyanalytic and one analytic function. That means that this function satisfies the equation (1), so if we find the operator derivative by $\bar{z}$ from $W$ and replace it in (1) we get:

$$
\begin{aligned}
& \frac{\hat{d} W}{d \bar{z}}=\frac{\hat{d}}{d \bar{z}}(\psi(z) \varphi(\bar{z}))=\psi(z) \cdot \frac{\hat{d} \varphi(\bar{z})}{d \bar{z}} \\
& \psi(z) \cdot \frac{\hat{d} \varphi(\bar{z})}{d \bar{z}}=A(\overline{\psi(z) \varphi(\bar{z}))}+B(\psi(z) \varphi(\bar{z}))+F \\
& \frac{1}{\bar{\psi}(\bar{z})} \cdot \frac{\hat{d} \varphi(\bar{z})}{d \bar{z}}=A \frac{\bar{\varphi}(z)}{\psi(z)}+B \frac{\varphi(\bar{z})}{\bar{\psi}(\bar{z})}+F \frac{1}{\psi(z) \bar{\psi}(\bar{z})}
\end{aligned}
$$

If we make one more transformation, we can get a proof to one more interesting statement. If we add on both sides the expression $-\frac{\varphi(\bar{z})}{\bar{\psi}^{2}(\bar{z})} \cdot \frac{\hat{d} \bar{\psi}(\bar{z})}{d \bar{z}}$ and given into consideration that

$$
\frac{\hat{d}}{d \bar{z}}\left(\frac{\varphi(\bar{z})}{\bar{\psi}(\bar{z})}\right)=\frac{1}{\bar{\psi}^{2}(\bar{z})}\left(\frac{\hat{d} \varphi(\bar{z})}{d \bar{z}} \cdot \bar{\psi}(\bar{z})-\varphi(\bar{z}) \frac{\hat{d} \bar{\psi}(\bar{z})}{d \bar{z}}\right)=\frac{1}{\bar{\psi}(\bar{z})} \frac{\hat{d} \varphi(\bar{z})}{d \bar{z}}-\frac{\varphi(\bar{z})}{\bar{\psi}^{2}(\bar{z})} \cdot \frac{\hat{d} \bar{\psi}(\bar{z})}{d \bar{z}}
$$

then, we get

$$
\frac{\hat{d}}{d \bar{z}}\left(\frac{\varphi(\bar{z})}{\bar{\psi}(\bar{z})}\right)=A \overline{\left(\frac{\varphi(\bar{z})}{\bar{\psi}(\bar{z})}\right)}+B \cdot \frac{\varphi(\bar{z})}{\bar{\psi}(\bar{z})}+F \frac{1}{\psi(z) \bar{\psi}(\bar{z})}-\frac{\varphi(\bar{z})}{\bar{\psi}^{2}(\bar{z})} \cdot \frac{\hat{d} \bar{\psi}(\bar{z})}{d \bar{z}}
$$

So, if

$$
\psi(z) \bar{\psi}(\bar{z})=1 \text { and }-\frac{\varphi(\bar{z})}{\bar{\psi}^{2}(\bar{z})} \cdot \frac{\hat{d} \bar{\psi}(\bar{z})}{d \bar{z}}=0
$$


we get another solution to the Vekua equation (1), i.e. the function $W_{1}=\frac{\varphi(\bar{z})}{\bar{\psi}(\bar{z})}$ which is not from the form (2). Again, (5) means that $\frac{\hat{d} \bar{\psi}(\bar{z})}{d \bar{z}}=0$.

So, now we can formulate the proven fact as a theorem.

Theorem 3. Let $\varphi=\varphi(\bar{z})$ be an antyanalytic function and $\psi=\psi(z)$ be an analytic function. If $W=\psi(z) \varphi(\bar{z})$ is a solution to the Vekua equation (1), then $W_{1}=\frac{\varphi(\bar{z})}{\bar{\psi}(\bar{z})}$ is also a solution to the Vekua equation (1), if the conditions (5) are satisfied.

Note. As we can see in all three cases, the second twin solution is not from the form (2). The functions are different, but the conditions are similar.

References

[1] Г. В. Колосов, Об одном приложении теории функции комплесного переменного к плоское задаче математическои упругости, 1909

[2] Г. Н. Положии, Обопштение теории аналитических фукции комплесного переменного, Издателство Киевского Университета, 1965

[3] D. S. Mitrinovic, Kompleksna analiza, Gragevinska knjiga,Beograd, 1971

[4] Д.Димитровски: Прилог кон теоријата на обопштените аналитички функции, Годишен зборник на ПМФ, Скопје, књ. 20, 1970

[5] М. Чанак, Неки доприноси теорији полианалитичких диференцијалних једначина, Математички весник, Београд, 2001

[6] S. Brsakoska, B. Ilievski, Two theorems for the Vekua equation, MASSEE International Congress on Mathematics MICOM 2009, 16-20.09.2009, Ohrid, Republic of Macedonia

Ss. Cyril and Methodius University

Faculty of Natural Sciences and Mathematics, Arhimedova 3, 1000 Skopje, R. Macedonia e-mail: sbrsakoska@gmail.com 